\title{
TỐI UUU HÓA VÙNG PHỦ SÓNG CỦA MẠNG CẢM BIẾN KHÔNG DÂY BẦNG THUẬT TOÁN VORONOI TRONG MÔI TRƯờnG 3D
}

\author{
Đặng Thanh Hải ${ }^{a^{*}}$, Lê Trọng Vĩnh ${ }^{b}$
}

\author{
${ }^{a}$ Khoa Công nghệ Thông Tin, Truờng Đại học Đà Lạt, Lâm Đồng, Việt Nam \\ ${ }^{b}$ Truoòng Đại học Khoa học Tư nhiên, Đại học Quốc gia Hà Nội, Hà Nội, Việt Nam \\ Nhận ngày 04 tháng 01 năm 2016 | Chấp nhận đăng ngày 16 tháng 03 năm 2016
}

\begin{abstract}
Tóm tắt
Trong nhũng năm gần đây mạng cảm biến không dây (WSN) được nhiều nhóm tác giả quan tâm. Một số phương pháp tối ưu hóa vùng phủ sóng của mạng cảm biến không dây được đề xuất để nâng cao hiệu quả triển khai mạng cảm biến do đó làm tăng độ phủ sóng, nhung hầu hết được xây dựng trên mô hình 2D, mà thường xa rời với thực tế. Trong bài báo này chúng tôi mở rộng thuật toán Voronoi để triển khai các cảm biến trong môi trường $3 D$ mà ở đó có nhiều vật cản làm ảnh hưởng đến khả năng phủ sóng của mạng cảm biến không dây.
\end{abstract}

Từ khóa: 3D; Mạng cảm biến không dây (WSN); Phủ sóng; Voronoi; Vật cản.

\section{GIỚI THIỆ}

Mạng cảm biến không dây (WSN) bao gồm một số các điểm cảm biến, các điểm cảm biến này có khả năng cảm nhận môi trường, thu thập dữ liệu, xử lý dữ liệu và giao tiếp với nhau. Dữ liệu sau đó được chuyển về trung tâm xử lý, phân tích tạo ra các thông tin hữu ích hỗ trợ ra quyết định của các chương trình ứng dụng khác nhau [1]. Hiện nay mạng cảm biến không dây được áp dụng trong nhiều lĩnh vực khác nhau: giám sát môi trường, cảnh báo cháy rừng, cảnh báo có sự tấn công, giám sát trong các địa hình phức tạp như trong lòng đại dương, hang động, các đường hầm trong mỏ,... Tùy thuộc vào loại ứng dụng mạng, cũng như bản chất và các điều kiện môi trường có ảnh hưởng đến hiệu quả và chi phí của mạng cảm biến không dây thì các kỹ thuật và phương pháp khác nhau được sử dụng để phát hiện và theo dõi các hiện tượng trong môi trường một cách hiệu quả.

\footnotetext{
*Tác giả liên hệ: Email: haidt@dlu.edu.vn
} 
Việc triển khai mạng cảm biến cần đảm bảo tối đa vùng phủ sóng nghĩa là phạm vi cần quan sát. Phạm vi phủ sóng đã được nghiên cứu rất kỹ trong môi trường $2 \mathrm{D}$ [1][2]. Gần đây, vấn đề này đã được mở rộng sang môi trường 3D vốn gần gũi với thực tế hơn cũng đã được nhiều nhà nghiên cứu quan tâm [3][4]. Tuy nhiên đây cũng là bài toán phức tạp vì trong môi trường thực tế tồn tại rất nhiều vấn đề ví dụ như có tòa nhà là chướng ngại vật ngăn cản việc phủ sóng của các cảm biến, có sông hồ mà ở đó không thể đặt được các cảm biến. Vậy mục tiêu đặt ra là tìm kiếm một giải pháp triển khai các cảm biến trong môi trường $3 \mathrm{D}$ đạt hiểu quả tức là các cảm biến không được đặt vào vùng cấm đồng thời phải đảm bảo khả năng phủ sóng của toàn mạng cảm biến hiệu quả nhất.

Một thuật toán Voronoi-Base được đề xuất triển khai trong môi trường $2 \mathrm{D}$ rất hiệu quả nhờ vào tính di chuyển được của các cảm biến [11]. Trong bài viết này chúng tôi đề xuất một cải tiến thuật toán Voronoi-Base để triển khai được các biến trong môi trường 3D với sự tồn tại của các chướng ngại vật mà vẫn đảm bảo được khả năng phủ sóng của toàn mạng cảm biến một cách hiệu quả. Phần còn lại của bài viết được tổ chức như sau. Phần 2 trình bày các phương pháp triển khai mạng cảm biến không dây. Phần 3 trình bày mô hình mạng cảm biến và thuật toán Voronoi cải tiến trong môi trường $3 \mathrm{D}$. Kết quả thực nghiệm sẽ được trình bày trong phần 4 . Cuối cùng, kết luận và hướng phát triển trong tương lai được đưa ra trong phần 5 .

\section{CÁC PHƯƠNG PHÁP TRIỀN KHAI MẠNG CẢM BIẾN}

\subsection{Mô hình phủ sóng của mạng cảm biến trong không gian $2 \mathrm{D}$ và $3 \mathrm{D}$}

Một mạng cảm biến được xem là có hiệu quả hay không, một trong các yếu được xem xét đó chính là khả năng thu thập thông tin và truyền dẫn dữ liệu về nút trung tâm để xử lý. Việc thu thập thông tin của mạng cảm biến phụ thuộc vào phạm vi phủ sóng cảm biến của toàn mạng đối với vùng mục tiêu và đặc biệt trong môi trường thực tế 3D có các vật cản làm ảnh hưởng đến phạm vi phủ sóng của mạng cảm biến.

Một mạng cảm biến không dây là một tập hợp các điểm cảm biến trong không gian Euclide, và mỗi điểm cảm biến có một phạm vi cảm nhận thông tin như Hình 1a. Mục tiêu là thiết kế phương án triển khai các điểm cảm biến trong không gian phủ sóng 
để đảm bảo vùng phủ sóng được nhiều nhất hay giảm thiểu không gian hố phủ sóng [5]. Hố phủ sóng là những vùng trong không gian cần phủ sóng mà không được bao phủ bởi bất kỳ một cảm biến nào của mạng cảm biến.

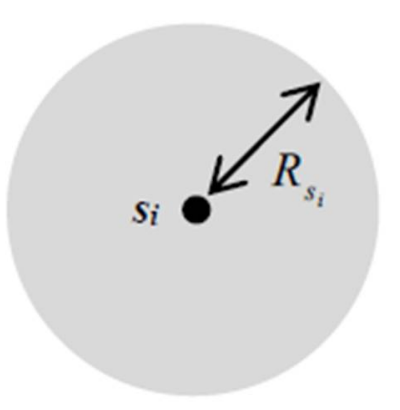

(a)

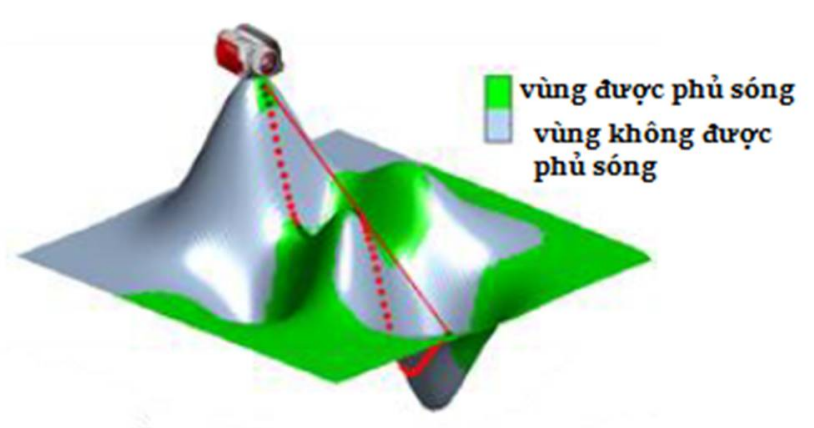

(b)

\section{Hình 1. Mô hình phủ sóng của cảm biến trong $2 \mathrm{D}$ và $3 \mathrm{D}$}

Các phương pháp tính toán khả năng phủ sóng của mạng cảm biến đã được đề xuất [6][7]. Đặc biệt đã có đã có những nghiên cứu mở rộng mô hình tính toán khả năng phủ sóng trong môi trường $3 \mathrm{D}$ có sử dụng khái niệm khả năng tầm nhìn giữa cảm biến và vùng cần phủ sóng (line of sights) như Hình 1b.[8][9].

\subsection{Các phương pháp tối ưu triển khai mạng cảm biến trong 2D}

Một số phương pháp tối ưu triển khai mạng cảm biến được đề xuất là phát hiện ra và làm giảm các hố phủ sóng do đó nó sẽ làm tăng vùng phủ sóng của các cảm biến trong mạng cảm biến không dây. Một trong các phương pháp tối ưu tăng cường khả năng phủ sóng của mạng cảm biến được tiếp cận dựa trên khái niệm về tính di động của các cảm biến. Các phương pháp này sử dụng cấu trúc hình học để phát hiện ra các hố phủ sóng rồi di chuyển các cảm biến để làm tăng vùng phủ sóng của toàn mạng, sơ đồ Voronoi và Delaunay Triangulation được ứng dụng vào phương pháp này và mang lại kết quả tốt [10][11].

Trong khái niệm sơ đồ Voronoi, ứng với mỗi điểm cảm biến sẽ xác định được một ô lưới Voronoi. Tất cả các điểm trong ô lưới của Voronoi được xem gần nhất với điểm cảm biến trong ô đó. Như vậy sau khi xây dựng xong sơ đồ Voronoi của tất cả cảm biến của mạng cảm biến không dây thì sẽ xác định được vùng phủ sóng của toàn 
mạng cảm biến như Hình 2. Nếu một điểm trong ô lưới Voronoi không được phủ sóng bởi điểm cảm biến của ô lưới đó thì nó cũng không được phủ sóng bởi bất cứ điểm cảm biến nào trong mạng và kết quả sinh ra các hố phủ sóng trong không gian cần phủ sóng của mạng cảm biến không dây.

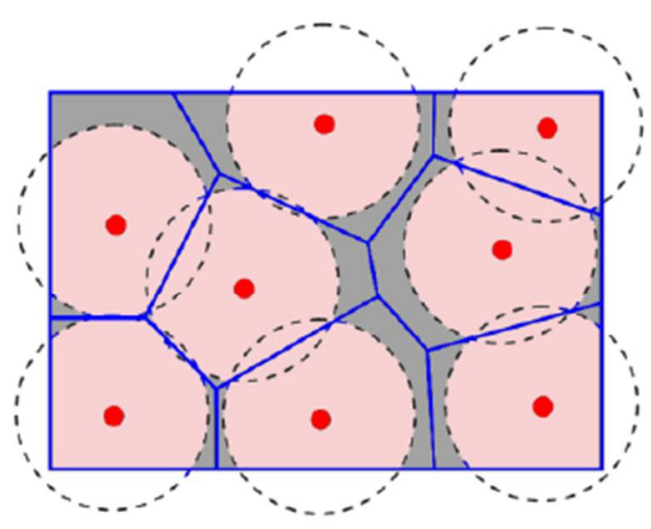

Hình 2. Các hố phủ sóng hình thành khi xây dụng sơ đồ Voronoi

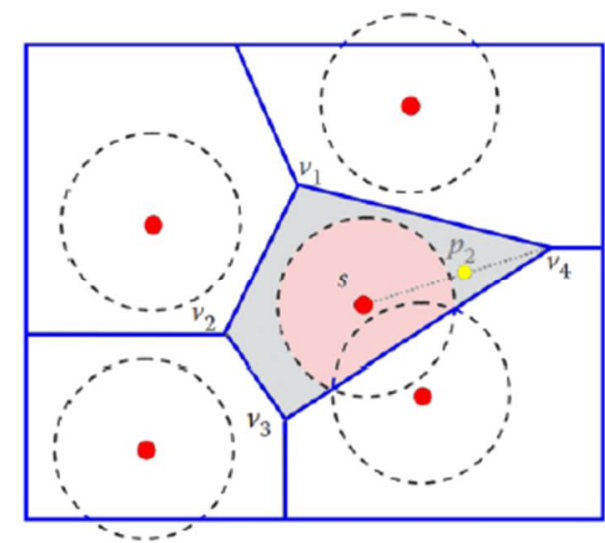

Hình 3. Chiến lược VOR đẩy các cảm biến để đạt độ phủ sóng tối đa

Nếu dựa vào tính di chuyển được của các cảm biến thì việc di chuyển này có khả năng phủ sóng được các hố phủ sóng đã nêu ở trên. Ba chiến lược di chuyển cảm biến trong sơ đồ Voronoi được đề xuất [11] đó là: Vector-Based (VEC), MiniMax, và Voronoi-Based (VOR). Tất cả các chiến lược này đều cải thiện tăng khả năng vùng phủ sóng của toàn mạng cảm biến bằng cách lặp đi lặp lại việc di chuyển các cảm biến trong mạng.

Thuật toán VOR là một chiến lược đẩy các cảm biến nội bộ trong ô lưới Voronoi về hướng có đỉnh xa nhất làm tăng khả năng phủ sóng của các cảm biến như Hình 3. Thuật toán VOR là thuật toán tham nên có thể làm giảm kích thước hố phủ sóng lớn nhất, nhưng sau mỗi lần di chuyển các cảm biến thì một hố phủ sóng mới có thể được tạo ra, mà hố phủ sóng này có thể bao phủ bởi việc di chuyển cảm biến ngược lại trong vòng lặp tiếp theo. Để khắc phục việc một cảm biến mới được di chuyển đi sau đó lại di chuyển trở lại trong lần lặp kế tiếp như đã thảo luận thì một điều kiện được bổ sung vào thuật toán là không cho phép bất cứ một cảm biến nào di chuyển ngược lại ngay lần lặp kế tiếp tức là trước khi một cảm biến di chuyển thuật toán kiểm tra quỹ đạo di chuyển của cảm biến có di chuyển trái ngược lại với lần lặp trước đó không? Nếu có thì việc di 
chuyển này sẽ dừng lại tại lần lặp này và hố phủ sóng đang đề cập sẽ được phủ sóng bởi cảm biến lân cận đó. Mặt khác việc di chuyển các cảm biến làm biến đổi hình dạng các ô lưới Voronoi mà có thể làm giảm diện tích phủ sóng của mạng cảm biến. Do đó thuật toán cần thêm điều kiện là khi di chuyển cảm biến đến các điểm mục tiêu thì cần phải đảm bảo làm tăng kích thước vùng phủ sóng trong từng ô lưới Voronoi và trong trường hợp điều kiện này không được đảm bảo thì vị trí mới của cảm biến chính là trung điểm giữa vị trí hiện hành và điểm mục tiên cần chuyển đến.

\section{MÔ HÌNH MẠNG CẢM BIẾN VÀ THUẬT TOÁN VORONOI CẢI TIẾN TRONG MÔI TRƯờNG 3D}

\subsection{Mô hình mạng cảm biến trong môi trường 3D}

Mô hình hóa một mạng cảm biến không dây trong môi trường 3D với các vật cản trong môi trường như sau:

- Gọi T là một địa hình DEM (Digital Elevation Model) là một ma trận mà các giá trị đại diện cho độ cao của điểm ô lưới.

○ cellsize : kích thước ô lưới

○ nrows, ncols : số hàng và số cột của ma trận

- $W S N=\left\{s_{1}, s_{2}, \ldots, s_{N}\right\}$ là một mạng cảm biến gồm $N$ các cảm biến $s_{j}$, $\forall j \in[1, N]$, là một bộ gồm các thành phần :

$$
s_{j}=\left\{x_{j}, y_{j}, h_{j}, r_{j}\right\}_{\forall j \in[1, N]}
$$

Trong đó:

○ $\left(x_{j}, y_{j}\right)$ là tọa độ của $s_{j}$ trong $\mathrm{Oxy}$.

○ $\quad h_{j}$ là độ cao của $s_{j}$ tại $\left(x_{j}, y_{j}\right)$

- $\quad r_{j}$ là bán kính của cảm biến $s_{j}$

- $\quad R=\left\{r_{1}, r_{2}, \ldots, r_{H}\right\}$ là tập các vật cản trong mô trường 
Trong đó:

$$
r_{i}=\left\{\left(x_{i}^{s}, y_{i}^{s}, h_{i}^{s}\right),\left(x_{i}^{f}, y_{i}^{f}, h_{i}^{f}\right)\right\}, \forall i \in[1,2, \ldots N]
$$

Mỗi vật cản được đại diện bởi tọa độ góc trên bên trái và góc dưới bên phải của một vùng

\subsection{Phương pháp Line-Of-Sight (LoS)}

Trong mô hình mạng cảm biến được đề xuất các cảm biến có khả năng di chuyển trong các ô lưới Voronoi, hơn nữa trong môi trường cũng giả định có các chướng ngại vật. Vậy để tránh được việc di chuyển của cảm biến vào các chương ngại vật thì trước tiên thuật toán phải kiểm tra giữa vị trí cảm biến với một đỉnh nào đó của ô lưới Voronoi có chướng ngại vật hay không? Phương pháp được áp dụng là LoS.

Các bước chính thực hiện của phương pháp LoS là trước tiên kẻ một đường thẳng giữa vị trí cảm biến đến một đỉnh của ô lưới Voronoi cần kiểm tra xem ở giữa có chướng ngại vật không, tiếp đến là chia đoạn thẳng này thành nhiều đoạn mỗi đoạn có kích thước bằng kích thước cellsize của ô lưới ma trận địa hình, các điểm chia này được lập thành một danh sách. Úng với mỗi điểm chia trong danh sách này được so sánh với độ cao trong địa hình. Trong bước này có thể khẳng định giữa hai điểm này có chướng ngại vật nếu như giá trị độ cao địa hình nằm ở phía trên đường thẳng như Hình 4.

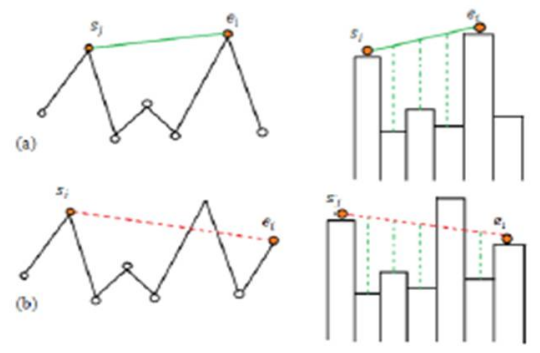

Hình 4. Phương pháp LoS

(a) Giữa hai điểm không cho chướng ngại vật

(b) Giữa hai điểm có chướng ngại vật

\subsection{Thuật toán Voronoi cải tiến trong môi trường 3D}

Thuật toán VOR có khả năng triển khai mạng cảm biến trong khu vực mục tiêu, tuy nhiên nó được cài đặt cho môi trường $2 \mathrm{D}$ bằng phẳng và không xem xét bất kỳ một 
rằng buộc nào về môi trường chẳng hạn như có các vật cản. Chúng tôi cải tiến thuật toán VOR có thể áp dụng triển khai cảm biến địa hình cho một mạng cảm biến không dây (WSN) trong môi trường 3D có các vật cản như định nghĩa trong công thức (2), trong ứng dụng này áp dụng phương pháp $\operatorname{LoS}$ để xác định các vật cản khi cần di chuyển các cảm biến. Thuật toán đề xuất chỉ những cảm biến mà có đỉnh xa nhất không được phủ sóng thì sẽ bị di chuyển: Nếu các đủnh của ô lưới Voronoi đã được phủ sóng bởi cảm biến thì cảm biến này không được di chuyển như Hình $5 b$, ngược lại, cảm biến sẽ di chuyển về hướng đỉnh xa nhất, trong trường hợp đỉnh xa nhất bị chắn bởi chướng ngại vật, chiều dài di chuyển của cảm biến được tính toán là khoảng cách từ cảm biến đến chướng ngại vật như Hình $5 \mathrm{c}$.

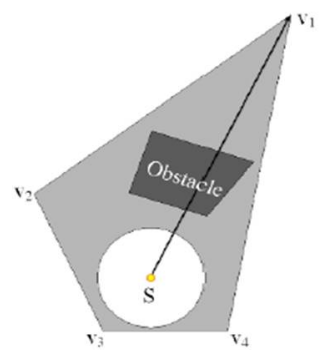

(a)

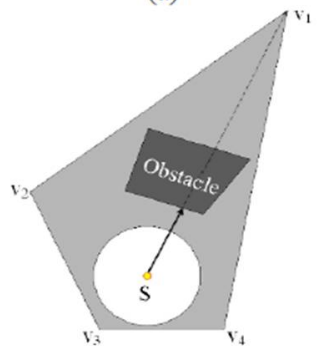

(c)

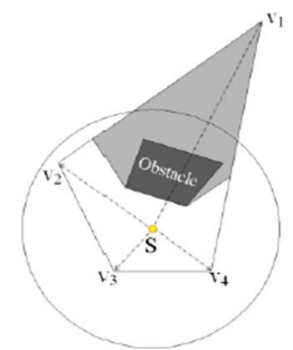

(b)

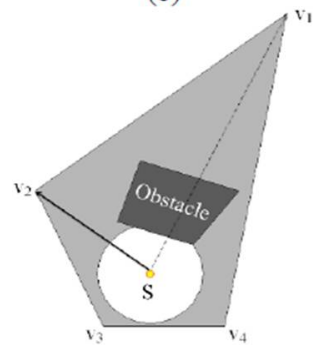

(d)

Hình 5. Thuật toán VOR cải tiến

(a) Cảm biến di chuyển về hướng đỉnh xa nhất mà chướng ngại vật không được xem xét.

(b) Tất cả các đỉnh của Voronoi (trừ $\mathrm{V}_{1}$ ) được phủ sóng bởi cảm biến, nên cảm biến sẽ không được di chuyển.

(c) Cảm biến di chuyển về hướng đỉnh xa nhất với khoảng cách giữa cảm biến và chướng ngại vật.

(d) Cảm biến di chuyển về hướng của đỉnh xa thứ hai vì không thể di chuyển tới đỉnh xa thứ nhất do chướng ngại vật.

Nếu cảm biến không thể di chuyển về hướng đỉnh xa nhất vì chướng ngại vật thì đỉnh xa thứ hai được chọn để cảm biến di chuyển về hướng của đỉnh này như Hình $5 \mathrm{~d}$.

\section{KẾT QUẢ THỰC NGHIỆM}

Thuật toán VOR cải tiến được áp dụng trong môi trường 3D như Hình 6 là một khu vực bằng phẳng chứa một tập các tòa nhà hình hộp. Mạng cảm biến bao gồm các 
cảm biến hình cầu và có bán kính phủ sóng $30 \mathrm{~m}$ được đề xuất. Ban đầu các cảm biến được triển khai với phân bố ngẫu nhiên trong không gian 3D có kích thước $200 \mathrm{~m} \mathrm{x}$ $200 \mathrm{~m}$.

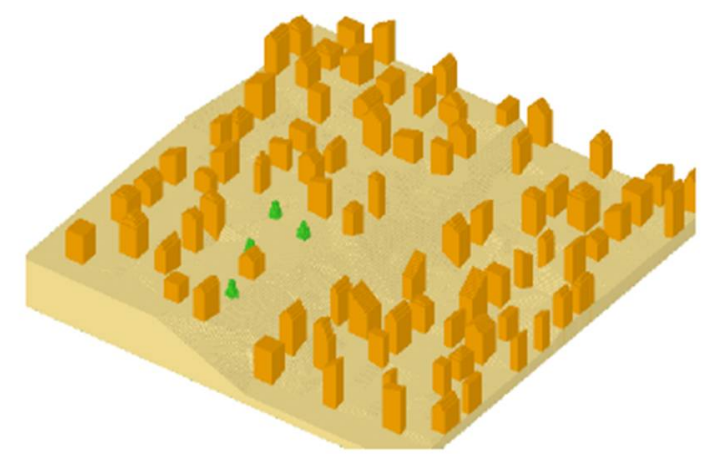

\section{Hình 6. Môi trường 3D với các tòa nhà hình khối}

Để đánh giá được sự ổn định của phương pháp đề xuất cả hai thuật toán VOR và VOR cải tiến được cài đặt và chạy thử nghiệm. Với số lần chạy là 30 , Bảng 1 cho biết tỉ lệ phần trăm phủ sóng của vùng mục tiêu là giá trị lớn nhất, nhỏ nhất và trung bình trong các lần chạy thử nghiệm. Kết quả trong phương pháp được đề xuất tốt hơn.

\section{Bảng 1. Kết quả thử nghiệm các thuật toán}

\begin{tabular}{llcc}
\hline & Thuật toán & VOR & VOR cải tiến \\
\hline \multirow{2}{*}{ Tỉ lệ phủ sóng } & Lớn nhất & 59.1811 & 77.7044 \\
& Nhỏ nhất & 39.9006 & 72.1707 \\
& Trung bình & 56.4122 & 75.4123 \\
\hline
\end{tabular}

\section{KẾT LUẬN VÀ HƯớNG PHÁT TRIỂN}

Bài viết đề xuất một phần cải tiến của thuật toán VOR để được áp dụng trong môi trường $3 \mathrm{D}$, điều này rất có ý nghĩa vì môi trường $3 \mathrm{D}$ là rất thực tế với các yếu môi trường đặc biệt trong môi trường luôn luôn tồn tại các chướng ngại vật. Trong ứng dụng cũng áp dụng kỹ thuật phân tích tầm nhìn LoS để phát hiện ra chướng ngại vật giữa cảm biến và các điểm mục tiêu cần xem xét. Kết quả thực hiện cho thuật toán VOR cải tiến cũng cho kết quả phủ sóng của cảm biến không dây một cách đáng kể. Tuy nhiên để khẳng định chắc chắn thuật toán VOR cải tiến đáp ứng tốt cho tất cả các địa hình trong môi trường $3 \mathrm{D}$ cần phải thực nghiệm trên nhiều địa hình phức tạp khác nhau mà tồn tại các chướng ngai vật là sông hồ,... và với kích thước địa hình lớn hơn. 


\section{TÀI LIỆU THAM KHẢO}

[1] Yick, J., Mukherjee, B., Ghosal, D., "Wireless sensor network survey," Computer networks, vol. 52, no. 12, 2008, pp. 2292-2330. (2008)

[2] Mulligan, R., Ammari, H.M., "Coverage in Wireless Sensor Networks". A Survey. Journal of Network Protocols and Algorithms 2(2), 27-53 (2010)

[3] Ravelomanana, V., "Extremal Properties of Three-Dimensional Sensor Networks with Applications". IEEE Transactions on Mobile Computing 3(3), 246-257 (2004).

[4] Huang, C., Tseng, Y., Lo, L., "The Coverage Problem in Three- Dimensional Wireless Sensor Networks". In: Proceedings of Global Telecommunications Conference (GLOBECOM 2004), November 29-December 3, pp. 3182-3186. IEEE (2004).

[5] Hossain, A., Biswas, P.K., Chakrabarti, S. Sensing Models and Its Impact on Network Coverage in Wireless Sensor Network, Industrial and Information Systems, 2008. ICIIS 2008. IEEE Region 10 and the Third international Conference on. IEEE, Kharagpur, pp. 1 - 5.(2008)

[6] Ghosh, A., Estimating Coverage Holes and Enhancing Coverage in Mixed Sensor Networks, Local Computer Networks, 2004. 29th Annual IEEE International Conference on, pp. 68 - 76. (2004)

[7] Wang, Y., Cao, G., Barrier Coverage in Camera Sensor Networks. MobiHoc '11 Proceedings of the Twelfth ACM International Symposium on Mobile Ad Hoc Networking and Computing. (2011).

[8] Akbarzadeh, V., Hung- Ren Ko, A., Gagn'e, C., Parizeau, M., Topography-Aware Sensor Deployment Optimization with CMA-ES, Parallel Problem Solving from Nature, PPSN XI. Springer, pp. 141-150. (2010).

[9] Argany, M., Mostafavi, M.A., Karimipour, F., Gagné, C., A gis based wireless sensor network coverage estimation and optimization: A voronoi ap-proach. Transacton on Computational Sciences Journal 14, 151-172. (2011).

[10] Argany, M., Mostafavi, M.A., Karimipour, F., Voronoi-based Approaches for Geosensor Networks Coverage Determination and Optimisation: A Survey, Proceedings of the 7th International Symposium on Voronoi Diagrams in Science and Engineering (ISVD 2010), Quebec, Canada, pp. 115-123. (2010).

[11] Karimipour, F., Argany, M., Mostafavi, M.A.,. Spatial coverage estimation and optimization in geosensor networks deployment, Wireless sensor networks: From theory to applications. CRC Press. (2013) 


\title{
OPTIMIZATION FOR THE COVERAGE PROBLEM OF WIRELESS SENSOR NETWORK WITH VORONOI ALGORITHM IN 3D ENVIRONMENTS
}

\author{
Dang Thanh Hai ${ }^{a^{*}}$, Le Trong Vinh \\ ${ }^{a}$ The Faculty of Information Technology, Dalat University, Lamdong, Vietnam \\ ${ }^{b}$ VNU University of Science, Hanoi, Vietnam \\ *Corresponding author: haidt@dlu.edu.vn \\ Article history \\ Received: January $04^{\text {th }}, 2016$ \\ Accepted: March 16 ${ }^{\text {th }}, 2016$
}

\begin{abstract}
In recent years, wireless sensor networks (WSN) has appeared interesting to many authors. Some methods to optimize the coverage of wireless sensor networks is proposed to improve the efficiency of deploying sensor networks, thus increasing the coverage; howerver, most are built on $2 D$ model, which are often hard to implement in reality. In this paper we extend Voronoi algorithm to deploy sensors in $3 D$ environments where there are obstacles which affect the ability of coverage of wireless sensor networks.
\end{abstract}

Keywords: Coverage; Voronoi; Obstacles; Wireless Sensor Networks. 